Running Head: LONGITUDINAL INVESTIGATION OF THE PATHWAYS MODEL

\title{
A Longitudinal Empirical Investigation of the Pathways Model of Problem Gambling
}

\author{
Youssef Allami $^{1,2}$, Frank Vitaro ${ }^{1,2}$, Mara Brendgen ${ }^{2,3}$, René Carbonneau ${ }^{1,2}$, \\ Éric Lacourse ${ }^{1}$, and Richard E. Tremblay ${ }^{1,2,4}$ \\ ${ }^{1}$ University of Montreal, Quebec Canada \\ ${ }^{2}$ GRIP, Quebec Canada \\ ${ }^{3}$ University of Quebec at Montreal, Quebec Canada \\ ${ }^{4}$ University College Dublin, Ireland
}

\begin{abstract}
Author Note
Youssef Allami, Department of Psychology and GRIP, University of Montreal, Quebec, Canada; Frank Vitaro, School of Psychoeducation and GRIP, University of Montreal, Quebec, Canada; Mara Brendgen, Department of Psychology and GRIP, University of Quebec at Montreal, Quebec, Canada; René Carbonneau, Department of Pediatrics and GRIP, University of Montreal, Quebec, Canada; Éric Lacourse, Department of Sociology, University of Montreal, Quebec, Canada; and Richard E. Tremblay, Department of Pediatrics, Department of Psychology and GRIP, University of Montreal, Quebec, Canada; School of Public Health, University College Dublin, Ireland.
\end{abstract}


Running Head: LONGITUDINAL INVESTIGATION OF THE PATHWAYS MODEL

Correspondence concerning this article should be addressed to Youssef Allami, Département de psychologie, Université de Montréal, Pavillon Marie-Victorin, C. P. 6128, succursale Centreville, Montréal (Québec) H3C 3J7, E-mail: youssef.allami@umontreal.ca

Compliance with Ethical Standards

Part of this study was funded by the Fonds de recherche du Québec sur la Société et la Culture. No conflict of interest is declared by the authors. All procedures performed in studies involving human participants were in accordance with the ethical standards of the institutional and/or national research committee and with the 1964 Helsinki declaration and its later amendments or comparable ethical standards

The final publication is available at Springer via https://dx.doi.org/10.1007/s10899-017-9682-6 


\begin{abstract}
Background and Aims: The Pathways Model of Problem Gambling suggests the existence of three developmental pathways to problem gambling, each differentiated by a set of predisposing biopsychosocial characteristics: Behaviorally Conditioned (BC), Emotionally Vulnerable (EV), and Biologically Vulnerable (BV) gamblers. This study examined the empirical validity of the Pathways Model among adolescents followed up to early adulthood. Design: A prospectivelongitudinal design was used, thus overcoming limitations of past studies that used concurrent or retrospective designs. Setting: Two samples were used: a) a population sample of French-speaking adolescents $(\mathrm{N}=1,033)$ living in low socio-economic status (SES) neighborhoods from the Greater Region of Montreal (Quebec, Canada), and b) a population sample of adolescents $(\mathrm{N}=3,017)$, representative of French-speaking students in Quebec. Participants: Only participants with at-risk or problem gambling by mid-adolescence or early adulthood were included in the main analysis $(n=180)$. Analyses: Latent Profile Analyses were conducted to identify the optimal number of profiles, in accordance with participants' scores on a set of variables prescribed by the Pathways Model and measured during early adolescence: depression, anxiety, impulsivity, hyperactivity, antisocial/aggressive behavior, and drug problems. Findings: A four-profile model fit the data best. Three profiles differed from each other in ways consistent with the Pathways Model (i.e., $\mathrm{BC}, \mathrm{EV}$, and BV gamblers). A fourth profile emerged, resembling a combination of EV and BV gamblers. Conclusions: Four profiles of at-risk and problem gamblers were identified. Three of these profiles closely resemble those suggested by the Pathways Model.
\end{abstract}

Abstract word count: 245

Keywords: gambling, adolescence, early adulthood, pathways model, longitudinal design 


\section{Introduction}

Problem-gambling (PG) becomes a disorder when a gambler exhibits a pre-set number of problems related to their gambling practice, from a legal, social, and self-control point of view (American Psychiatric Association 2013). The prevalence of PG among the general adult population varies between 2-5\%, depending on the country studied (Williams et al. 2012; Shaffer and Hall 2001). This rate is higher among adolescents and young adults (between 4-7\%; Shaffer and Hall 2001; Villella et al. 2011).

Although variable-oriented research has regularly identified the same set of risk factors for PG (e.g., impulsivity, hyperactivity, emotional problems, delinquency, or drug problems), some contradictory findings have also emerged. For example, impulsivity has been extensively documented as predicting PG (Dussault et al. 2011; Johansson et al. 2009; Nower et al. 2004). However, some researchers have also failed to find a link (Dannon et al. 2010; Langewisch and Frisch 1998). Seemingly contradictory results in the literature may be due to the erroneous practice of combining all problem gamblers into a single group. There is also accumulating evidence that young problem gamblers, like their adult counterparts, are not a homogenous group (Gupta et al. 2012; Milosevic and Ledgerwood 2010; Nower et al. 2013).

\section{The Pathways Model}

Many researchers and clinicians have devised different classifications of gamblers (Blaszczynski and Nower 2002; Moran 1970; Sharpe 2002). However, in Milosevic and Ledgerwood's (2010) analysis of 17 different studies that have classified PG into different subgroups, the authors concluded that a three-group model - resembling the three pathways proposed by the Pathways Model (Blaszczynski and Nower 2002) - appeared most consistently. The Pathways Model proposes three developmental pathways to PG, each differentiated by a set 
of pre-disposing risk factors and consequences from gambling: Biologically Vulnerable (BV), Emotionally Vulnerable (EV), and Behaviorally Conditioned (BC) gamblers.

BV problem gamblers display biopsychosocial vulnerabilities. These gamblers exhibit a range of behaviors typically associated with impulse-control disorders, which have been found to be partly under genetic influence and related to specific neuro-cognitive deficits (Dussault et al. 2011; Lobo and Kennedy 2009; Nower et al. 2004). They also demonstrate high levels of antisocial or aggressive behaviors unrelated to gambling, as well as multiple drug use. This subgroup exhibits the most severe levels of PG, and tends to start gambling the earliest. They also tend to persist in their gambling habits.

EV gamblers suffer from an underlying affective dysregulation. Negative family background experiences or past trauma partially contribute to their emotional vulnerability. PG develops in response to their affective state, as a means of emotional regulation. In line with this subgroup, depression has been concurrently and predictively linked to PG (Dussault et al. 2011; Jacobs 2005). Members of this subgroup may also display other maladaptive behaviors (e.g., alcohol abuse) to cope with their emotional difficulties. Their problem gambling levels are fairly stable, but less severe than BV gamblers.

Finally, BC gamblers do not show signs of any biological or affective predisposition. They start gambling for reasons of excitement and socialization (i.e., peer pressure), but fluctuate between light and heavy problem gambling because of conditioning (i.e., differential reinforcement due to gains and losses). Affective problems (e.g., depressive symptomatology) that may be associated with their gambling are understood as a consequence, rather than a precedent, of their gambling. They experience fewer gambling problems than their EV and BV counterparts, and tend to start 
gambling the latest. As a group, they also tend to be the first to desist from gambling once they enter into adult roles during early adulthood, but exceptions may exist.

\section{Empirical support for the model}

A number of studies have tested the Pathways Model with adult samples (Valleur et al. 2015; Nower et al. 2013). For instance, in a study among 581 problem gamblers who participated in the US National Epidemiologic Survey on Alcohol and Related Conditions, Nower et al. (2013) identified three profiles evocative of the subtypes covered by the Pathways Model. In contrast, only one study has examined the applicability of this model to an adolescent population (Gupta et al. 2012). This study identified five subgroups of problem gamblers $(n=109)$, three of which resembled those described by the Pathways Model. However, all validation studies so far used a cross-sectional design (i.e. all measures collected at the same time). This approach makes it impossible to distinguish co-occurring mental health conditions that precede problem gambling from those that accompany it. For example, in the case of EV gamblers, a cross-sectional approach cannot help establish whether emotional problems preceded their gambling problems. To address this shortcoming and to achieve a stronger empirical validation of the Pathways Model, a longitudinal design - that collects measures unique to each pathway prior to the emergence of PG - must be used.

\section{Study Objectives}

The first aim of this study was to identify the optimal number of PG profiles in terms of personal characteristics (i.e., impulsivity, hyperactivity, anxiety, depressive symptoms, antisocial/aggressive behavior, and drug problems) in early adolescence before the emergence of problem gambling. The second aim was to validate the empirically derived profiles by contrasting them with regards to age of onset of gambling behavior, and other gambling characteristics (i.e., 
number of gambling problems and frequency of gambling behavior) by mid adolescence (age 16) and early adulthood (age 23). Two samples from Quebec (Canada), both of which used a longitudinal design, were used. The first sample ("Sample A") consists of low socioeconomic status (SES) French-speaking adolescent males (high-risk group). The second sample (“Sample B”) is representative of all Quebec students attending francophone schools. Gambling problems were measured in both samples when participants were 16 and 23 years old. Personality (i.e., impulsivity, hyperactivity, depression, anxiety, and antisocial/aggressive behavior) correlates were collected at age 12 using teacher ratings. Teacher ratings were chosen over parent ratings because the former have been found to better predict problematic behavior in children (Power et al. 1998; Verhulst et al. 1994). Drug problems were assessed using a self-report measure at age 14. Selfreports were chosen because young adolescents are likely to hide their drug use from their teachers, resulting in an underestimation of drug problems from the teacher's perspective. Moreover, surveys in Quebec have shown that adolescents generally start developing significant drug problems around the age of 14 years (Dubé et al. 2009). Given that the two samples were merged to maximize the number of problem gamblers who would be included in the analyses, we also examined whether the results varied from one sample to the other.

Although we used two population-based samples, only at-risk or problematic gamblers were selected for this study. Since the Pathways Model serves to identify developmental pathways leading to PG, it makes sense to only target participants who have developed some levels of gambling problems. Past studies that have examined the Pathways model used a similar strategy.

\section{Hypotheses}

As outlined by the Pathways Model, we expected to find one subgroup (i.e., BV) with higher levels of impulsivity, hyperactivity, drug problems, and antisocial/aggressive behavior during 
early adolescence relative to all other subgroups. Another subgroup (i.e., EV) was expected to display higher levels of depression and anxiety during early adolescence, relative to all other groups. Also, we expected to find a third and final subgroup (i.e., BC) with low levels on all indicator variables, compared to the other two subgroups. Finally, we expected BV gamblers to report a higher number of gambling problems both during late adolescence and early adulthood and an earlier age of onset of gambling behavior compared to EV gamblers, who in turn were expected to report more gambling problems and an earlier age of onset of gambling behavior than BC gamblers.

\section{Method}

\section{Participants}

Sample A. Participants were part of an ongoing longitudinal study that started in 1984 with 1,033 French-speaking kindergarten boys attending schools in economically disadvantaged areas in Montreal, Quebec, Canada. Participants included in the initial sample represented $87 \%$ of all boys attending the 53 schools that participated in the study. Data used in this study were collected when the participants were $12,14,16$ and 23 years old. This non-probabilistic convenience sample represents a high-risk group (i.e., low SES males).

Sample B. Participants were part of the ongoing Québec Longitudinal Study of Kindergarten Children, a representative sample of the French-speaking children attending kindergarten in the province of Quebec, Canada, in 1986-1987. From an initial pool of 6,397 six-year old children, 2,000 participants were selected among the children for whom both parent reports and teacher reports were available, to ensure good participation during follow-up. Out of all the parents and teachers initially contacted, $63 \%$ of parents and $68 \%$ of teachers provided complete reports. An additional 1,017 participants with elevated scores on a disruptive behavior scale (Tremblay et al. 
1991) were included to provide sufficient statistical power when examining problematic behavior.

The final, partly probabilistic, partly high risk, sample consisted of 3,017 participants (58\% male).

See Rouquette et al. (2014) for a complete explanation of sampling procedure. Data used in this study were collected when the participants were $12,14,16$ and 23 years old.

\section{Procedure}

The study was approved by the School Board and active written consent was obtained from parents in both samples. All participants actively provided their assent to take part in the study. Participants were also informed before data collection that their answers were strictly confidential and that they could end their participation at any time. All measures were administered in the Spring towards the end of the school year. Unless otherwise stated, the same instruments were administered in both samples.

\section{Measures}

Teacher Ratings. When participants were 12 years old, teachers completed the Social Behavior Questionnaire (Tremblay et al. 1987). Each item was rated on a 3-point scale (0: Does not apply, 1: Applies sometimes, and 2: Frequently applies). From this instrument, items were selected to assess Impulsivity, Hyperactivity, Depression, Anxiety, and Antisocial/Aggressive behavior. Item scores were summed to obtain a total score for each variable.

Impulsivity. A total of 7 items (e.g. "acts without thinking”) were included in this scale (Sample A: Cronbach's $\alpha=.89$; Sample B: $\alpha=.88$ ). Higher scores indicate higher levels of impulsivity. Total scores varied between 0 and 13 .

Hyperactivity. A total of 3 items (e.g. "squirmy, fidgety child") were included in this scale (Sample A: $\alpha=.86$; Sample B: $\alpha=.85$ ). Higher scores indicate higher levels of hyperactivity. Total scores varied between 0 and 4. 
Depression. A total of 4 items (e.g. “appears miserable, unhappy, tearful or distressed”) were included in this scale (Sample A: $\alpha=.62$; Sample B: $\alpha=.63$ ). Higher scores indicate higher levels of depression. Total scores varied between 0 and 7.

Anxiety. A total of 3 items (e.g. "is worried about many things") were included in this scale (Sample A: $\alpha=.86$; Sample B: $\alpha=.84$ ). Higher scores indicate higher levels of anxiety. Total scores varied between 0 and 6 .

Antisocial/Aggressive behavior. A total of 9 items (e.g. "has stolen things on one or more occasions.") were included in this scale (Sample A: $\alpha=.87$; Sample B: $\alpha=.87$ ). Higher scores indicate higher levels of antisocial/aggressive tendencies. Total scores varied between 0 and 16 .

Self-Reported Ratings. Participants reported on their drug and alcohol-related problems when they were 14 years old. They also reported on their gambling-related problems when they were 16 and 23 years old.

Drug and Alcohol Problems. The Personal Experience Screen Questionnaire (PESQ; Henly and Winters 1988) was used to gauge illicit drug and alcohol problems. This test examines 18 potential problems (e.g. "When you have consumed alcohol or drugs, how many times have you seen, felt, or heard things that were not really there?"), with item scores ranging from 1 (never) to 4 (often). Higher rates indicate more severe levels of drug and alcohol problems. Total scores varied between 18 and 43 (Sample A: $\alpha=.93$; Sample B: $\alpha=.90$ ). The following three items were included to detect if participants were lying: "In order to pay for alcohol or drugs, how many times have you conned people with counterfeit money?", "How many times have you consumed alcohol or other drugs (including cigarettes and marijuana) while parachuting?", and "How many times have you obtained alcohol or drugs from a police officer?" If a participant responded positively to one of these questions, the overall score was deleted and treated as missing data. 
Gambling Problems and Gambling Frequency. In accordance with recommendations made by Nower and Blaszczynski (2005), we used adolescent and adult versions of the South Oaks Gambling Screen to assess gambling problems (SOGS-RA at age 16 years and SOGS at age 23 years; Lesieur and Blume 1987; Winters et al. 1993). The SOGS is a self-reported questionnaire designed to assess problems related to past-year gambling practices. The SOGS-Revised for Adolescents (SOGS-RA) comprises 12 Yes/No items, whereas the adult version consists of 20 items. Both instruments share 11 items. However, the twelfth item in the adolescent version (i.e., "borrowing money to gamble or to pay gambling debts") is split into nine items in the adult version, with each source of borrowing added up independently (e.g., borrowing from family and friends would result in a score of 2 in the adult version, and a score of 1 in the adolescent version). An additional nine items were used to analyze the frequency (0: never, 1: less than once a month, 2: once a month, 3: once per week, 4: daily) at which participants engaged in a variety of gambling activities (e.g., play cards for money). A frequency score was computed by adding scores for each gambling activity.

The SOGS-RA has been validated for screening gambling problems among adolescents aged 14 to 20 years old (Chiesi et al. 2013), and the SOGS has been validated in a variety of settings (i.e., Gamblers Anonymous, university students, and hospital employees; Lesieur \& Blume, 1987). In Sample A, Cronbach's $\alpha$ was .76 and .88 at ages 16 and 23, respectively. In Sample B, Cronbach's $\alpha$ was .76 and .75 at ages 16 and 23, respectively.

In Sample A, age of onset of gambling was prospectively assessed starting at age 12 years, by asking participants if they had gambled in the past year. In Sample B, age of onset was retrospectively assessed at age 23 by asking participants at what age they first gambled. 


\section{Results}

\section{Data Treatment and Preliminary Analyses}

Sample A. Of the initial sample of 1,033 participants, 669 (65\%) and $504(49 \%)$ had valid data on gambling at ages 16 and 23, respectively. Among those with gambling data for at least one time-point $(n=801), 84(10.5 \%)$ displayed at-risk levels of gambling at one or more time-points and were included in our PG classification: $55(8.2 \%)$ had two or more problems at ages 16 and $32(6.2 \%)$ had four or more problems at age 23 . Two or more gambling problems in adolescence corresponds to Level 2 of the Levels terminology used by Shaffer and Hall (2001; i.e., at-risk or transitional gambling), whereas 4 or more gambling problems in early adulthood corresponds to one less problem than that required to be classified as a "probable pathological gambler" by the SOGS (i.e., problem gambler). These criteria were similar to those used by Gupta et al. (2012) and by Nower et al. (2013).

Sample B. Of the initial sample of 3,017 participants, 1,798 (60\%) and 1,445 (48\%) had valid data on gambling at ages 16 and 23, respectively. Among those with gambling data for at least one time-point $(\mathrm{n}=2248), 114(5 \%)$ displayed at-risk levels of gambling at one or more time-points and were included in our PG classification: $101(5.6 \%)$ had two or more problems at age 16 and $16(1.1 \%)$ had four or more problems at age 23.

Considering that the main goal of the study was to identify antecedents of PG, and that PG may emerge in adolescence or adulthood, problem gamblers identified at either age period (i.e., midadolescence or early adulthood) from both samples were combined for all analyses. This strategy also allowed us to maximize sample size and hence boost statistical power. When conducted on gambling data at ages 16 and 23 years, Little's (1988) test was not significant, $\chi^{2}(2, \mathrm{~N}=4050)=$ 4.062, $p=.131$, indicating that gambling data were Missing Completely At Random (MCAR). 
Therefore, cases with missing data could be safely ignored when selecting our subset of problemgamblers without introducing much bias.

Preliminary analyses were conducted to check the distributional properties of our data. Univariate outliers were winsorized (Tabachnick and Fidell 2012). Considering many variables showed a skewed distribution, we used a Maximum Likelihood estimation with Robust standard errors (MLR; B. Muthén and Asparouhov 2002); an estimation method specifically conceived for non-normally distributed data.

Of the 198 participants selected, 18 had missing data on all indicator variables at ages 12 or 14 and were excluded from the PG classification. Of the remaining 180 participants, 21 were female ( $21.4 \%$ of Sample B; $11.7 \%$ of the combined samples), $11 \%$ had missing data on the teacher-rated scales at age 12 ; and $26 \%$ had missing data on the self-rated scale of drug abuse at age 14 years (including 6 participants whose scores were deleted because they failed the built-in lie-detecting items). Missing data was handled using the Full Information Maximum Likelihood method for the Latent Profile Analysis (see below), and using Multiple Imputation (10 iterations) for subsequent analyses. These methods are appropriate when data is missing completely at random, which was the case according to Little's test (Little 1988), $\chi^{2}(6, \mathrm{~N}=180)=7.91, p=.245$. Table 1 summarizes descriptive statistics for the main variables used in this study, after combining problem gamblers from Samples A and B.

\section{Latent Profile Analysis}

Latent Profile Analysis (LPA; Collins and Lanza 2010) is a type of Latent Class Analysis, a set of statistical tools that allow identification of profiles according to a set of continuous indicator variables (DiStefano 2012). The number of profiles is not known beforehand and the optimal solution is found by using a set of model fit indicators, combined with maximum-likelihood tests 
(probability-based classification). Following Celeux and Soromenho (1996) and Nylund et al. (2007), the following fit indicators were used: Bayesian information criterion (BIC), Entropy, and Lo-Mendell-Rubin Adjusted Likelihood Ratio Test (LMR-ALRT). The empirically-derived profiles were further validated by testing for differences between profiles across all indicator variables using a MANOVA. LPA was performed, using a step-wise mixture modeling technique with MPlus 6.12 (L. K. Muthén and Muthén 2010), to identify the optimal number of PG profiles based on the following indicator variables suggested by the Pathways Model: teacher-rated impulsivity, depression, anxiety, hyperactivity, and antisocial/aggressive behavior at age 12; self-reported drug problems at age 14.

The LPA was conducted with the 180 participants who displayed at-risk or problematic levels of gambling either at 16 or at 23 years of age. Model fit indices are reported in Table 2. Two- and three-class models separated groups based on their degree of severity across all variables (i.e., high on all vs. low on all). Furthermore, although the five- and six-class models had lower BIC scores, this indicator kept decreasing no matter how many classes were added. The tendency for the BIC to consistently decrease has been observed across many other studies that have used continuous indicators, rather than categorical, to determine the optimal number of classes (Nower et al. 2013; Marsh et al. 2009; Geiser et al. 2014). Therefore, in accordance with Marsh et al.'s (2009) recommendations, the best-fitting solution was selected based on qualitative and quantitative changes between the classes. A four-class model turned out to be optimal, as demonstrated by the statistically significant $L M R-A L R T$, higher entropy, and profiles that were qualitatively meaningful (i.e., not simply separated into high, medium, low levels across all variables).

Three of the four profiles closely resembled the BC $(n=105 ; 58 \%), \mathrm{EV}(n=31 ; 17 \%)$, and BV $(n=36 ; 20 \%)$ pathways. The fourth profile $(n=8 ; 5 \%)$ had high levels on all indicator variables, 
and resembled an overlap between EV and BV profiles, hereafter dubbed Bio-Emotionally Vulnerable (BEV) gamblers; a similar profile was also found by Gupta et al., 2012).

\section{Confirming PG Profiles}

All subsequent analyses were conducted with SPSS version 22 (IBM 2013). Participants were classified into one of the four profiles identified, based on their most likely class membership. Participants classified into the fourth pathway were not included in these analyses because of the low participant count $(n=8)$, resulting in a subset of 172 problem gamblers. First, a chi-square test of independence was conducted to test equality of gender distribution across gambling groups. The test was not statistically significant, $\chi^{2}(2, \mathrm{~N}=172)=2.04, p=.360$. Second, a MANOVA was conducted to validate the distinctiveness of all profiles identified. All six indicator variables (i.e., Impulsivity, Depression, Anxiety, Hyperactivity, Antisocial/Aggressive Behavior, and Drug Problems) were included as dependent variables. Drug Problems was the only variable to show excessively high skewness and kurtosis levels, and was therefore subjected to a logarithmic transformation prior to analyses.

Profiles served as a fixed factor (i.e., BC, EV, and BV). The results from the MANOVA indicated a significant overall difference between groups across indicator variables: average Pillai's Trace $=1.13, F(12,330)=35.61, p<.001$, partial eta squared $=.56$, power to detect the effect was 1.00. The results remained significant after controlling for Gender and SES. Priority was given to Pillai's Trace because it has been shown to be very robust to violations of MANOVA assumptions (Scheiner 2001), such as heteroscedasticity. Levene's test of equality of error variances yielded satisfying results for all variables except Antisocial/Aggressive, $F(2,169)=$ 7.32, $p<.001$, Hyperactivity, $F(2,169)=3.07, p=.049$, and Drug Problems, $F(2,169)=4.19$, $p=.017$. Univariate $F$ tests for between-subject effects revealed a significant difference between 
groups for: Impulsivity, $F(2,169)=106.05, p<.001$; Hyperactivity, $F(2,169)=108.50, p<$ .001 ; Depression, $F(2,169)=71.01, p<.001$; Anxiety, $F(2,169)=38.44, p<.001$; Antisocial/Aggressive Behavior, $F(2,169)=36.62, p<.001$; and Drug Problems, $F(2,169)=$ $5.35, p=.006$. A second MANOVA also included Sample (A or B) as a second factor in order to test for a Pathway * Sample interaction, which was not significant, Pillai's Trace $=0.09, F(12$, $324)=1.32, p=.205$. Therefore, the differences between pathways were not due to belonging to one sample or another.

Post hoc tests were conducted to examine specific differences between groups. The StudentNewman-Keuls test (appropriate for unequal group sizes) was used for this purpose. Table 3 summarizes mean scores of all variables for each group. Some significant differences $(\alpha=.05)$, consistent with the Pathways Model, are particularly worth noting:

- EV gamblers were rated by their teacher as manifesting more depressive symptoms than BV gamblers, who in turn had higher levels than BC gamblers;

- EV gamblers were rated by their teachers as manifesting more anxiety symptoms than BV gamblers, who in turn had higher levels than BC gamblers;

- BV gamblers were rated by their teachers as manifesting more impulsive symptoms than EV and BC gamblers;

- BV gamblers were rated by their teachers as manifesting more hyperactivity symptoms than EV and BC gamblers;

- BV gamblers were rated by their teachers as manifesting more antisocial/aggressive behaviors symptoms than EV and BC gamblers; and

- BV gamblers reported more drug problems compared to EV and BC gamblers. 


\section{Validating PG Profiles}

Gambler profiles (i.e., BC, EV, and BV) were then compared in terms of their gambling practices (Age of Onset, Frequency, and Problems; mean scores are reported in Table 3). First, a series of ANOVAs were conducted. No difference between groups appeared with regards to Age of Onset, $F(2,169)=.76, p=.469$. Likewise, no differences were found with regards to Gambling Frequency at both time-points: age $16, F(2,169)=1.40, p=.249$; age $23, F(2,169)=0.92, p=$ .400 .

The comparison for gambling problems required a different analytical strategy since gambling problems are count data. A choice had to be made between a Poisson and a negative binomial regression model. As there was a high level of overdispersion in the data (i.e. variance 2 to 3 times greater than the mean, for both time-points), a negative binomial regression model was estimated (Gardner et al. 1995). BV gamblers were set as a reference group, and served as a basis for comparison with the two other groups (i.e., BV vs. BC, BV vs. EV). SES and gender were included as covariates.

At age 16 years, the omnibus likelihood ratio $\chi^{2}$ test revealed no effects of the independent variables: $\chi^{2}(4, N=172)=2.31, p=.679$, indicating that there was no difference between pathways. At age 23, however, a significant effect was found: $\chi^{2}(4, N=172)=21.49, p<.001$. The gender covariate was significant $(p=.001)$, indicating that males had approximately 1.27 more problems than females. Above and beyond the significant effect of the gender covariate, BV gamblers had approximately $.48(p=.045)$ more gambling problems compared to their $\mathrm{BC}$ counterparts, and .66 more gambling problems compared to EV gamblers $(p=.053)$. When controlling for gambling problems at age 16, the only remaining difference was between BV and BC gamblers $(p=.033)$ 
Finally, considering that participants were selected based on whether or not they displayed PG at either age 16 or 23 years (i.e., could have displayed PG at age 16 but not 23, or vice-versa), another series of chi-square tests of independence were conducted to test whether PG prevalence differed between pathways at each time-point. The test was not significant at age $16, \chi^{2}(2, \mathrm{~N}=$ $172)=1.87, p=.393$. However, results were significant at age $23, \chi^{2}(2, \mathrm{~N}=172)=6.78, p=.034$. In other words, among problem-gamblers at age 16, the proportion belonging to each profile was similar to the initial percentages identified through the LPA. However, at age 23 years, the proportion belonging to the BV subgroup increased (i.e., they were over-represented), the proportion of BC gamblers decreased (i.e., they were under-represented), and the proportion of EV gamblers remained stable.

\section{Discussion}

The Pathways Model is a developmental etiological model with a set of predisposing factors that can lead at-risk gamblers to follow one pathway over another. The current study is the first to examine the model's empirical validity for adolescents using a longitudinal design that can disentangle the chronological ordering between predisposing factors and gambling profiles.

In line with the first study objective, four profiles of problem gamblers were identified based on personal characteristics measured in early adolescence, three of which closely resemble the subtypes described by the Pathways Model: Behaviourally Conditioned (58\%), Emotionally Vulnerable (17\%), and Biologically Vulnerable problem gamblers (20\%), and a fourth sub-type displaying an overlap of Biologically and Emotionally Vulnerable (BEV) characteristics (5\%). These findings are partially concordant with those reported by Gupta et al. (2012) and Nower et al. (2013). BC, EV, and BV profiles were also identified in both of these studies. The BEV profile 
was only identified in the Gupta et al. (2012) study, which examined adolescent gambling, suggesting that this profile may be specific to this developmental period.

As hypothesized, EV gamblers displayed higher levels of anxiety and depressive symptoms compared to all other groups. Also, BV gamblers were more impulsive and hyperactive than their EV and BC counterparts. However, contrary to the validation studies by Gupta et al. (2012) and Nower et al. (2013), a hyperactivity component was not present in EV gamblers. Nonetheless, this result is concordant with the Pathways model. With regards to drug problems, our results are also consistent with the Pathways Model: BV gamblers displayed higher scores compared to BC and EV gamblers, who did not differ from each other.

With regards to the second study objective (i.e., comparing profiles in terms of gambling behavior), the three pathways identified in this study did not differ with regards to age of onset of gambling behavior. This conflicts with theoretical predictions, considering that BV gamblers are hypothesized to start gambling earlier and to gamble more frequently than their $\mathrm{EV}$ and $\mathrm{BC}$ counterparts. Results with respect of age of onset, however, could have been different had we started assessing gambling behavior before the age of 12 , as some children may have started gambling at an earlier age (Vitaro and Wanner 2011). Moreover, no differences were found between groups at age 16 with regards to gambling problems on the SOGS-RA, possibly because age 16 corresponds to the peak of gambling problems for all the groups (Wanner et al. 2006). However, at age $23, \mathrm{BV}$ gamblers reported more gambling problems compared to their $\mathrm{BC}$ counterparts, which is in line with theoretical predictions. Also, BV gamblers' problems seemed to persist, whereas EV and BC gamblers seemed to desist, as evidenced by BV gamblers' higher rate of $P G$ at age 23 . 
By showing that characteristics correlate differently with specific pathways, the present results also help reconcile contradictory findings found in past studies. For instance, Dannon et al.'s (2010) finding that PG was not associated with impulsivity could be due to the fact that their sample consisted mainly of BC and EV gamblers. Furthermore, McCormick et al.'s (1984) finding that depression does not always precede gambling problems might be explained by the qualitative difference between $\mathrm{BC}$ and EV gamblers. This does not mean, however, that personal characteristics are the only risk factors associated with problem gambling. Other risk factors include environmental as well as structural factors (Johansson et al. 2009).

Our findings support the need to adopt a differentiated view with respect to etiological models of adolescent PG. More specifically, our findings suggest that different personality characteristics may conspire to put some individuals at risk for problem gambling, although the origins of these characteristics and their possible interplay with socio-environmental factors remain unknown. Our findings also point to the need to shift toward a differential targeted prevention approach in regard to PG in adolescents. Considering that disordered gambling has recently been classified among other substance use disorders (American Psychiatric Association 2013), prevention program developers should find inspiration from existing targeted prevention programs that have focused on substance use by targeting the specific personal and socio-environmental risk factors experienced by different subgroups (Conrod et al. 2013; Allami and Vitaro 2015).

\section{Strengths and Limitations}

This study has several strengths. First, adopting a longitudinal perspective allowed us to disentangle personal characteristics generally associated with PG from the specific gambling profiles they are linked to. Second, we used different raters to assess participants' characteristics and gambling problems, thus reducing bias that could result from shared method variance. Third, 
we included longitudinal measures of gambling characteristics to further validate the gambling profiles. Despite these assets, some limitations must be noted. First, even with our large combined sample, the relatively small sample size of problem gamblers may have limited our ability to effectively identify differences between groups. For example, the fourth profile of PG could have been included in subsequent analyses had a larger sample been used. Statistical significance might also have been achieved on comparisons of gambling frequency, problems and gender. Second, there was a relatively large amount of missing data. Although handled with state of the art statistical techniques, this may limit the generalizability of our results. Third, the instruments used to assess personality predispositions (i.e., impulsivity, hyperactivity, depression, anxiety, and antisocial/aggressive behavior) were relatively short, with the number of items per scale ranging from 2 to 7, and may only be considered as screening tools, rather than thorough diagnostic assessments. Finally, although most measures were collected prospectively, some were collected using a retrospective strategy (i.e., age of onset for Sample B) and may thus have been affected by recall bias.

Despite these limitations, the current study showed that four profiles of adolescent at-risk and problem gamblers can be empirically derived, three of which closely resemble those suggested by the Pathways Model, thereby contributing to the accumulating evidence supporting the validity of this theoretical model. 
Table 1

Descriptive Statistics (Problem Gamblers, $N=180$ )

\begin{tabular}{lcccr}
\hline Variable (Age) & $M$ & $S D$ & Skewness & Kurtosis \\
\hline Antisocial/Aggressive behavior (12) & 3.21 & 3.83 & 1.30 & .97 \\
Impulsivity (12) & 3.39 & 3.60 & .92 & -.25 \\
Hyperactivity (12) & 1.30 & 1.60 & 1.07 & -.02 \\
Anxiety (12) & 2.03 & 1.60 & .40 & -.68 \\
Depression (12) & 1.45 & 1.66 & 1.25 & 1.32 \\
Drug problems (14) & 20.31 & 5.20 & 3.56 & 14.89 \\
$\quad$ & .61 & .93 & 1.37 & .75 \\
Log(Drug Problems) & & & & \\
Gambling problems (16) & 2.86 & 2.06 & 1.52 & 4.14 \\
Gambling frequency (16) & 6.52 & 4.39 & 1.13 & 2.36 \\
Gambling problems (23) & 1.81 & 3.09 & 1.80 & 2.25 \\
Gambling frequency (23) & & & & \\
Age of Gambling Onset & 3.82 & 3.54 & 1.72 & 3.54 \\
\hline
\end{tabular}

Note. M: Mean, SD: Standard deviation. 
Table 2

Model Fit Indicators for Latent Profile Analysis $(N=180)$

\begin{tabular}{lccc}
\hline Model & BIC $^{\mathrm{a}}$ & Entropy & $\begin{array}{c}\text { LMR-ALRT }^{\mathrm{b}} \\
(p \text { value })\end{array}$ \\
\hline 2-class & 4347 & .847 & .000 \\
3-class & 4260 & .864 & .063 \\
4-class & $\mathbf{4 1 6 8}$ & .874 &. $\mathbf{0 3 7}$ \\
5-class & 4142 & .863 & .523 \\
6-class & 4111 & .868 & .438 \\
\hline
\end{tabular}

Note. Best-fitting model is shown in bold.

${ }^{a}$ Bayesian Information Criterion

${ }^{\mathrm{b}}$ Lo-Mendell-Rubin Adjusted Likelihood Ratio Test 
Table 3

Group Means (M) and Standard Deviations (SD) for Behaviorally Conditioned (BC), Emotionally Vulnerable (EV), and Biologically Vulnerable (BV) Gamblers, Averaged Across 10 Imputations

\begin{tabular}{|c|c|c|c|c|c|c|}
\hline \multirow[b]{2}{*}{ Variable (Age) } & \multicolumn{2}{|c|}{$\mathrm{BC}(n=105)$} & \multicolumn{2}{|c|}{$\mathrm{EV}(n=31)$} & \multicolumn{2}{|c|}{$\mathrm{BV}(n=36)$} \\
\hline & $M$ & $S D$ & $M$ & $S D$ & $M$ & $S D$ \\
\hline Antisocial/Aggressive & $2.02^{c}$ & 2.77 & $2.42^{c}$ & 2.36 & $6.91^{\mathrm{a}, \mathrm{b}}$ & 3.98 \\
\hline behavior (12) & & & & & & \\
\hline Impulsivity (12) & $2.07^{\mathrm{c}}$ & 2.26 & $1.81^{\mathrm{c}}$ & 1.77 & $8.25^{\mathrm{a}, \mathrm{b}}$ & 2.52 \\
\hline Hyperactivity (12) & $.75^{c}$ & .99 & $.52^{\mathrm{c}}$ & .76 & $3.40^{\mathrm{a}, \mathrm{b}}$ & 1.15 \\
\hline Anxiety (12) & $1.33^{\mathrm{b}, \mathrm{c}}$ & 1.24 & $3.42^{\mathrm{a}, \mathrm{c}}$ & 1.48 & $2.65^{\mathrm{a}, \mathrm{b}}$ & 1.13 \\
\hline Depression (12) & $.75^{\mathrm{b}, \mathrm{c}}$ & 1.06 & $3.29^{\mathrm{a}, \mathrm{c}}$ & .81 & $1.36^{\mathrm{a}, \mathrm{b}}$ & 1.08 \\
\hline Drug Problems (14) & $20.23^{c}$ & 3.78 & $19.85^{c}$ & 2.98 & $23.31^{\mathrm{a}, \mathrm{b}}$ & 7.69 \\
\hline Gambling Problems (16) & 2.63 & 1.97 & 3.45 & 2.25 & 2.78 & 2.12 \\
\hline Gambling Frequency (16) & 6.21 & 4.30 & 7.58 & 4.36 & 6.99 & 4.77 \\
\hline Gambling Problems (23) & $1.73^{c}$ & 3.05 & 1.31 & 2.19 & $2.93^{\mathrm{a}}$ & 3.35 \\
\hline Gambling Frequency (23) & 3.81 & 3.20 & 3.96 & 3.20 & 3.70 & 3.09 \\
\hline Age of gambling onset & 12.45 & 3.58 & 12.32 & 3.01 & 12.75 & 2.95 \\
\hline
\end{tabular}

\footnotetext{
${ }^{\mathrm{a}}$ Different from BC group at the $\alpha=.05$ level.

${ }^{\mathrm{b}}$ Different from EV group at the $\alpha=.05$ level.

${ }^{\mathrm{c}}$ Different from BV group at the $\alpha=.05$ level.
} 


\section{References}

Allami, Y., \& Vitaro, F. (2015). Pathways model to Problem Gambling: Clinical Implications for Treatment and Prevention Among Adolescents. Canadian Journal of Addiction, 6(2), 1319.

American Psychiatric Association (2013). Diagnostic and Statistical Manual of Mental Disorders: DSM-5. Arlington, VA: American Psychiatric Publishing Incorporated.

Blaszczynski, A., \& Nower, L. (2002). A pathways model of problem and pathological gambling. Addiction, 97(5), 487-499.

Celeux, G., \& Soromenho, G. (1996). An entropy criterion for assessing the number of clusters in a mixture model. Journal of Classification, 13(2), 195-212, doi:10.1007/BF01246098.

Chiesi, F., Donati, M. A., Galli, S., \& Primi, C. (2013). The suitability of the South Oaks Gambling Screen-Revised for Adolescents (SOGS-RA) as a screening tool: IRT-based evidence. Psychology of Addictive Behaviors, 27(1), 287-293, doi:10.1037/a0029987.

Collins, L. M., \& Lanza, S. T. (2010). Repeated-Measures Latent Class Analysis and Latent Transition Analysis. In Latent Class and Latent Transition Analysis (pp. 179-224): John Wiley \& Sons, Inc.

Conrod, P. J., O’Leary-Barrett, M., \& Newton, N. (2013). Effectiveness of a selective, personalitytargeted prevention program for adolescent alcohol use and misuse: A cluster randomized controlled trial. JAMA Psychiatry, 70(3), 334-342, doi:10.1001/jamapsychiatry.2013.651.

Dannon, P. N., Shoenfeld, N., Rosenberg, O., Kertzman, S., \& Kotler, M. (2010). Pathological gambling: an impulse control disorder? Measurement of impulsivity using neurocognitive tests. Israel Medical Association Journal, 12(4), 243-248. 
DiStefano, C. (2012). Cluster analysis and latent class clustering techniques. In Handbook of developmental research methods (pp. 645-666). New York, NY: The Guilford Press.

Dubé, G., Bordeleau, M., Cazale, L., Fournier, C., Traoré, I., Plante, N., et al. (2009). Enquête québécoise sur le tabac, l'alcool, la drogue et le jeu chez les élèves du secondaire,

2008. (pp. 155). Québec: Institut de la statistique du Québec.

Dussault, F., Brendgen, M., Vitaro, F., Wanner, B., \& Tremblay, R. E. (2011). Longitudinal links between impulsivity, gambling problems and depressive symptoms: a transactional model from adolescence to early adulthood. Journal of Child Psychology and Psychiatry, 52(2), 130-138, doi:10.1111/j.1469-7610.2010.02313.x.

Gardner, W., Mulvey, E. P., \& Shaw, E. C. (1995). Regression analyses of counts and rates: Poisson, overdispersed Poisson, and negative binomial models. Psychological Bulletin, 118(3), 392-404, doi:10.1037/0033-2909.118.3.392.

Geiser, C., Okun, M. A., \& Grano, C. (2014). Who is motivated to volunteer? A latent profile analysis linking volunteer motivation to frequency of volunteering. Psychological Test and Assessment Modeling, 56(1), 3-24.

Gupta, R., Nower, L., Derevensky, J. L., Blaszczynski, A., Faregh, N., \& Temcheff, C. (2012). Problem Gambling in Adolescents: An Examination of the Pathways Model. Journal of Gambling Studies, doi:10.1007/s10899-012-9322-0.

Henly, G. A., \& Winters, K. C. (1988). Development of Problem Severity Scales for the Assessment of Adolescent Alcohol and Drug Abuse. Substance Use \& Misuse, 23(1), 6585, doi:10.3109/10826088809027491.

IBM (2013). IBM SPSS Statistics for Windows. (22 ed.). Armonk, NY: IBM Corp. 
Jacobs, D. F. (2005). Youth Gambling in North America. In J. Derevensky, \& R. Gupta (Eds.), Gambling Problems in Youth (pp. 1-24): Springer US.

Johansson, A., Grant, J. E., Kim, S. W., Odlaug, B. L., \& Gotestam, K. G. (2009). Risk factors for problematic gambling: a critical literature review. Journal of Gambling Studies, 25(1), 6792, doi:10.1007/s10899-008-9088-6.

Langewisch, M. J., \& Frisch, G. R. (1998). Gambling Behavior and Pathology in Relation to Impulsivity, Sensation Seeking, and Risky Behavior in Male College Students. Journal of Gambling Studies, 14(3), 245-262, doi:10.1023/A:1022005625498.

Lesieur, H. R., \& Blume, S. B. (1987). The South Oaks Gambling Screen (SOGS): a new instrument for the identification of pathological gamblers. American journal of Psychiatry, 144(9), 1184-1188.

Little, R. J. A. (1988). A Test of Missing Completely at Random for Multivariate Data with Missing Values. Journal of the American Statistical Association, 83(404), 1198-1202, doi: $10.2307 / 2290157$.

Lobo, D. S. S., \& Kennedy, J. L. (2009). Genetic aspects of pathological gambling: a complex disorder with shared genetic vulnerabilities. Addiction, 104(9), 1454-1465, doi:10.1111/j.1360-0443.2009.02671.x.

Marsh, H. W., Lüdtke, O., Trautwein, U., \& Morin, A. J. (2009). Classical latent profile analysis of academic self-concept dimensions: Synergy of person-and variable-centered approaches to theoretical models of self-concept. Structural equation modeling, 16(2), 191-225.

McCormick, R. A., Russo, A. M., Ramirez, L. F., \& Taber, J. I. (1984). Affective disorders among pathological gamblers seeking treatment. American journal of Psychiatry, 141(2), 215218. 
Milosevic, A., \& Ledgerwood, D. M. (2010). The subtyping of pathological gambling: A comprehensive review. Clin Psychol Rev, 30(8), 988-998, doi:10.1016/j.cpr.2010.06.013.

Moran, E. (1970). Varieties of pathological gambling. British Journal of Psychiatry, 116(535), 593-597.

Muthén, B., \& Asparouhov, T. (2002). Using Mplus Monte Carlo Simulations In Practice: A Note On Non-Normal Missing Data In Latent Variable Models. http://www.statmodel.com/download/webnotes/mc2.pdf. Accessed 5 juillet 20142014.

Muthén, L. K., \& Muthén, B. O. (2010). Mplus User's Guide. (6 ed., Vol. Sixth Edition). Los Angeles, CA: Muthén \& Muthén.

Nower, L., \& Blaszczynski, A. (2005). A Pathways Approach to Treating Youth Gamblers. In J. Derevensky, \& R. Gupta (Eds.), Gambling Problems in Youth: Theoretical and Applied Perspectives (pp. 189-209): Springer US.

Nower, L., Derevensky, J. L., \& Gupta, R. (2004). The relationship of impulsivity, sensation seeking, coping, and substance use in youth gamblers. Psychology of Addictive Behaviors, 18(1), 49-55, doi:10.1037/0893-164x.18.1.49.

Nower, L., Martins, S. S., Lin, K.-H., \& Blanco, C. (2013). Subtypes of disordered gamblers: Results from the National Epidemiologic Survey on Alcohol and Related Conditions. [Peer Reviewed]. Addiction, .108(4), pp, doi:10.1111/add.12012 23072599.

Nylund, K. L., Asparouhov, T., \& Muthén, B. O. (2007). Deciding on the number of classes in latent class analysis and growth mixture modeling: A Monte Carlo simulation study. Structural equation modeling, 14(4), 535-569.

Power, T. J., Doherty, B. J., Panichelli-Mindel, S. M., Karustis, J. L., Eiraldi, R. B., Anastopoulos, A. D., et al. (1998). The Predictive Validity of Parent and Teacher Reports of ADHD 
Symptoms. Journal of Psychopathology and Behavioral Assessment, 20(1), 57-81, doi:10.1023/A:1023035426642.

Rouquette, A., Côté, S. M., Pryor, L. E., Carbonneau, R., Vitaro, F., \& Tremblay, R. E. (2014). Cohort Profile: The Quebec Longitudinal Study of Kindergarten Children (QLSKC). International Journal of Epidemiology, 43(1), 23-33, doi:10.1093/ije/dys 177.

Scheiner, S. M. (2001). MANOVA: Mutliple response variables and multispecies interactions. In S. M. Scheiner, \& J. Gurevitch (Eds.), Design and analysis of ecological experiments (pp. 94-112). USA: Oxford University Press.

Shaffer, H. J., \& Hall, M. N. (2001). Updating and refining prevalence estimates of disordered gambling behaviour in the United States and Canada. Revue canadienne de santé publique, 92(3).

Sharpe, L. (2002). A reformulated cognitive-behavioral model of problem gambling. A biopsychosocial perspective. Clin Psychol Rev, 22(1), 1-25.

Tabachnick, B. G., \& Fidell, L. S. (2012). Cleaning Up Your Act: Screening Data Prior to Analysis. In Using Multivariate Statistics: Pearson Education.

Tremblay, R. E., Desmarais-Gervais, L., Gagnon, C., \& Charlebois, P. (1987). The Preschool Behaviour Questionnaire: Stability of its Factor Structure Between Cultures, Sexes, Ages and Socioeconomic Classes. International Journal of Behavioral Development, 10(4), 467484, doi:10.1177/016502548701000406.

Valleur, M., Codina, I., Vénisse, J.-L., Romo, L., Magalon, D., Fatséas, M., et al. (2015). Towards a Validation of the Three Pathways Model of Pathological Gambling. Journal of Gambling Studies, 1-15, doi:10.1007/s10899-015-9545-y. 
Verhulst, F. C., Koot, H. M., \& Van der Ende, J. (1994). Differential predictive value of parents' and teachers' reports of children's problem behaviors: A longitudinal study. Journal of Abnormal Child Psychology, 22(5), 531-546.

Villella, C., Martinotti, G., Di Nicola, M., Cassano, M., La Torre, G., Gliubizzi, M. D., et al. (2011). Behavioural Addictions in Adolescents and Young Adults: Results from a Prevalence Study. Journal of Gambling Studies, 27(2), 203-214, doi:10.1007/s10899-0109206-0.

Vitaro, F., \& Wanner, B. (2011). Predicting early gambling in children. Psychology of Addictive Behaviors, 25(1), 118.

Wanner, B., Vitaro, F., Ladouceur, R., Brendgen, M., \& Tremblay, R. E. (2006). Joint trajectories of gambling, alcohol and marijuana use during adolescence: a person- and variablecentered developmental approach. Addict Behav, 31(4), 566-580, doi:10.1016/j.addbeh.2005.05.037.

Williams, R. J., Volberg, R. A., \& Stevens, R. M. (2012). The population prevalence of problem gambling: Methodological influences, standardized rates, jurisdictional differences, and worldwide trends. Ontario Problem Gambling Research Centre.

Winters, K. C., Stinchfield, R. D., \& Fulkerson, J. (1993). Toward the development of an adolescent gambling problem severity scale. Journal of Gambling Studies, 9(1), 63-84, doi:10.1007/BF01019925. 